\title{
Esqueletização multiescala para análise de forma de agregados
}

\author{
Lilian Tais de Gouveia ${ }^{1}$, Fernanda Elisa Stelle ${ }^{2}$, Luciano José Senger ${ }^{3}$, Edson Aparecido Martins Filho ${ }^{4}$
}

\begin{abstract}
Resumo: A forma dos agregados minerais afeta várias propriedades das misturas asfálticas, dentre elas a resistência à deformação permanente e à fadiga. A forma está diretamente relacionada com as proporções geométricas dos agregados. Vários são os métodos laboratoriais utilizados para determinar a forma dos agregados, mas frequentemente eles são trabalhosos e, por vezes, subjetivos. Neste trabalho, é apresentado o método de esqueletização multiescala para determinação das características de forma dos agregados. Este método é simples de ser executado, e os resultados são de fácil entendimento. Neste método, as partículas são reduzidas a uma estrutura mais simples, chamada de esqueleto, em diferentes níveis de detalhamento (limiares). Dois tipos de agregados foram utilizados, basalto e seixo, com graus de esfericidade de 0,77 e 0,87 , respectivamente, segundo a tabela Rittenhouse. As imagens dos agregados foram obtidas através de um scanner. Os esqueletos das imagens dos agregados foram obtidos utilizando a transformada distância, e os modelos de classificação foram obtidos a partir dos esqueletos por meio dos descritores de forma: raios médios, variância, número de ramos, tamanho do maior ramo e esfericidade. O melhor nível de detalhamento do esqueleto foi obtido empregando o valor de limiar igual a 5. O modelo de classificação apresentou $97 \%$ de acurácia quando empregado utilizando o algoritmo IBk. Este elevado índice de acerto demonstra a robustez do método de esqueletização para a determinação e a classificação dos agregados quanto à forma.
\end{abstract}

Palavras-chave: esqueletização multiescala, forma, agregados.

\begin{abstract}
Mineral aggregates' shape affect various properties of asphalt mixtures, such as resistance to permanent deformation and to fatigue. Shape is directly related to geometric proportions of aggregates. There are several laboratory methods used to determine aggregates' shapes, but they are often cumbersome and sometimes subjective. In this paper, the multiscale skeleton method for determining the shape characteristics of aggregates is presented. This method is simple to be applied and the results are easy to understand. In this method, the particles are reduced to a simpler structure, called the skeleton, at different levels of detail (thresholds). Two types of aggregates were studied, basalt and gravel, with degrees of sphericity 0.77 and 0.87 , respectively, according to the Rittenhouse table. Images of aggregates were obtained by using a digital scanner. The skeletons of images of aggregates were obtained using the distance transform and knowledge was extracted from skeletons by shape descriptors: average radii, variance, number of branches, largest branch and sphericity. The best level of detail of the skeleton (threshold) was equal to 5, which allows the classification algorithm to achieve an accuracy of $97 \%$. This satisfactory rate of success demonstrates the robustness of the multiscale skeleton method for the determination and classification of aggregates shapes.
\end{abstract}

Keywords: mulscale skleton, shape, aggregates.

\section{INTRODUÇÃO}

O desempenho dos pavimentos asfálticos depende também das propriedades e das proporções dos principais componentes constituintes do revestimento, que são os agregados, o cimento asfáltico e o volume de vazios. Os agregados constituem mais de $90 \%$ do volume total das misturas asfálticas e têm como função principal formar seu esqueleto estrutural, responsável por resistir ao movimento dos veículos. O esqueleto estrutural é formado pelas partículas de agregados de vários tamanhos com características de forma, de angularidade e de textura adequadas. Esqueletos estruturais resistentes à deformação permanente são geralmente compostos por agregados com formas cúbicas, cantos angulares e textura superficial rugosa. Quanto mais arredondado e liso for o agregado, menor deve ser o intertravamento entre suas partículas, portanto, menor também

\footnotetext{
1 Lilian Tais de Gouveia, Departamento de Engenharia Civil, Universidade Estadual de Ponta Grossa. (Itgouveia@gmail.com)

2 Fernanda Elisa Stelle, Departamento de Engenharia Civil, Universidade

Estadual de Ponta Grossa. (fernanda_stelle@hotmail.com)

${ }^{3}$ Luciano José Senger, Departamento de Informática, Universidade

Estadual de Ponta Grossa. (Isenger@uepg.br)

${ }^{4}$ Edson Aparecido Martins Filho, Departamento de Informática,

Universidade Estadual de Ponta Grossa. (edinho_999@hotmail.com)

Manuscrito recebido em 13/052014 e aprovado para publicação em 30/04/2015.

Este artigo é parte de TRANSPORTES v. 23, n. 2, 2015. ISSN: 2237-1346 (online). DOI:10.14295/transportes.v23i2.788
}

deve ser a resistência à deformação permanente da mistura asfáltica formada (Cross e Brown, 1992; Kim et al., 1992; Gouveia, 2006). Muitos dos defeitos dos pavimentos podem estar diretamente relacionados à escolha inadequada dos agregados, que devido às suas características de forma passam a formar esqueletos estruturais muito rígidos ou muito deformáveis o que pode vir a causar problemas como trincas por fadiga e deformações permanentes (Gouveia et al., 2007).

Segundo Suguio (1973), a esfericidade é uma grandeza que tenta expressar numericamente o grau de aproximação da forma de uma partícula qualquer com a de uma esfera perfeita. Os agregados são materiais naturais e heterogêneos que podem apresentar elevada variabilidade e magnitude do conjunto forma/angularidade. Portanto, a classificação de suas características de forma não é uma tarefa fácil, tendo em vista que um mesmo tipo de agregado pode apresentar formas bem distintas.

Agregados que sofrem processo de britagem tendem a ter, dependendo de sua origem, características similares de fissuras ou de fragmentação. Já agregados não britados, como areias e seixos, sofrem processos diferentes de modelagem de suas formas, dependendo do processo de desgaste a que foram submetidos. Por exemplo, o seixo extraído de rios tende a ter suas formas mais arredondadas devido ao desgaste pela água. Dessa maneira, as características de forma dos agregados estão também relacionadas aos pro- 
cessos mecânicos ou naturais de desgaste e/ou de fragmentação sofridos, além das características próprias de cada material.

Frazão (2002) classifica a forma dos agregados em cúbica (ou esférica), lamelar (ou achatada, ou discóide), alongada (ou prismática) e alongada-lamelar. Uma partícula é chamada de lamelar ou achatada quando sua espessura é relativamente pequena em relação às outras dimensões, e alongada quando seu comprimento é relativamente maior em relação às outras dimensões.

Para uso em concreto asfáltico, a forma ideal dos agregados é a cúbica, pois agregados com partículas achatadas e alongadas não propiciam boa trabalhabilidade à mistura asfáltica. Além disso, durante a compactação, essas partículas podem quebrar, alterando a composição granulométrica da mistura e a forma das partículas. Essas partículas tendem a arranjar-se apoiando faces de maior estabilidade (faces planas), resultando em menores vazios no agregado mineral. Outro inconveniente é que partículas planas e/ou alongadas apresentam maior superfície específica se comparadas às partículas de forma cúbica, levando a consumos maiores de ligante asfáltico.

Os métodos laboratoriais destinados à determinação da forma dos agregados utilizam equipamentos simples, porém, muitas vezes os ensaios são trabalhosos, demorados e subjetivos. Atualmente, vários métodos computacionais com base em análise de imagens vêm sendo propostos, afim de avaliar características importantes dos agregados de maneira mais direta e objetiva. Dentre estes, incluem-se as abordagens baseadas na Transformada de Hough (Wilson e Klotz, 1996); morfologia fractal para avaliar a angularidade do agregado miúdo (Masad et al., 2000); decomposição Wavelet em escala de cinza para análise da textura superficial utilizando imagens binárias para caracterização da forma do agregado (Chandan et al. 2004); e análise de Fourier aplicada aos perfis das partículas de agregados para determinar a angularidade (Bowman et al., 2001). Além desses, pode-se citar o método de curvatura multiescala para análise de forma e de angularidade dos agregados graúdos e miúdos (Gouveia et al., 2010), método de entropia para a determinação do grau de alteração de agregados de basalto (Gouveia et al., 2011 e Gouveia et al., 2013) e sistemas automáticos de caracterização dos agregados quanto à sua geometria tais como, o Blob3D (Ketcham e Shashidhar, 2001), o qual analisa quantitativamente as características de misturas asfálticas através de imagens tomográficas com raio X; e o Aggregate Imaging Measurement System (AIMS) (Fletcheret al. 2002), que apresenta um mecanismo de aquisição de imagens e um software que analisa a forma e a angularidade de agregados graúdos e miúdos. Também outras características importantes para a caracterização de agregados como resistência ao polimento e degradação (Mahmoud e Masad, 2007; Bessa et al., 2014) e classificação do estado de alteração utilizando redes neurais (Senger e Gouveia, 2010) foram avaliadas utilizando métodos de processamento de imagens.

Nesta pesquisa, é proposto um método para classificação da forma dos agregados de maneira rápida e objetiva, utilizando esqueletização multiescala. A proposta de utilização de um scanner para obtenção das imagens permite, por ser um equipamento simples, de custo acessível e disponível em vários laboratórios, que as imagens sejam obtidas de maneira simples e com qualidade adequada. No método proposto, a forma da partícula do agregado é reduzida a uma estrutura mais simples, chamada de esqueleto. O esqueleto que traduz a forma pode ser representado com mais ou menos detalhes. O detalhamento é ajustado por meio da escolha de um valor de limiar. O esqueleto é obtido de maneira rápida empregando algoritmos computacionais implantado no ambiente de programação Scilab. O scilab é um ambiente de programação com código livre e distribuição gratuita. Esse software científico foi desenvolvido para computação numérica, semelhante ao Matlab, e fornece bibliotecas e ferramentas para aplicações científicas. Obtidos os esqueletos, esses são analisados através de descritores de forma, por exemplo, a quantidade de ramos formados nos esqueletos. A análise das imagens por descritores também é realizada com a utilização de algoritmos implantado no Scilab.

\section{ESQUELETIZAÇÃO MULTIESCALA DE IMAGENS DIGITAIS}

Uma imagem digital é definida como uma função bidimensional $\mathrm{f}(x, y)$, representada por meio de matriz, composta de um número finito de elementos chamados pixels, em que x e y são coordenadas espaciais, e a amplitude de $\mathrm{f}$ em qualquer par de coordenadas $(x, y)$ é chamada de intensidade ou nível de cinza da imagem. Cada elemento da matriz corresponde a um pixel da imagem, com localização e valor específico (Gonzalez e Woods, 2010).

A forma de um objeto é uma informação visual importante e tem sido utilizada como uma das características básicas para descrever o conteúdo de imagens. Uma abordagem para a representação da forma de um objeto é reduzilo a uma estrutura mais simples, chamada de esqueleto, que pode ser obtido através de técnicas, como transformada do eixo médio, transformada distância, diagrama de Voronoi e afinamento de objetos (Costa e Cesar Jr., 2001).

A transformada distância, técnica utilizada neste trabalho para obtenção dos esqueletos, corresponde ao mapa resultante da distância entre cada ponto interior do objeto e sua borda mais próxima (Pedrini e Schwartz, 2008). A distância entre os pontos de uma imagem pode ser calculada com diferentes métricas. Uma métrica muito utilizada em processamento de imagens, e também neste trabalho, é a euclidiana. Gonzalez e Woods (2010) definem a distância euclidiana entre p e q, cujas coordenadas são $(x, y)$ e $(s, t)$, como: $(\mathrm{p}, \mathrm{q})=\sqrt{(\mathrm{x}-\mathrm{s})^{2}+(\mathrm{y}-\mathrm{t})^{2}}$. Após aplicar esta transformada a todos os pontos do objeto, o esqueleto será composto pelos pontos que possuem maiores valores de distâncias da borda.

Na Figura 1, estão ilustradas imagens da sequência de processamento das imagens até obtenção dos esqueletos. A Figura 1 (a) representa uma imagem do agregado de basalto utilizado, a (b) representa sua imagem binária, a (c) ilustra a imagem da transformada distância, onde o esqueleto da imagem, formado pelos valores mais altos de distância do eixo com relação à borda (regiões mais claras), formarão os esqueletos com diferentes níveis de detalhamento (número de ramos), em função do valor de limiar utilizado. O esqueleto pode ser constituído de maiores ou menores níveis de detalhamento em função dos valores de limiares adotados, numa abordagem multiescala. A variação de limiares permite encontrar um nível de detalhamento satisfa- 


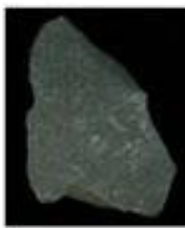

(a)

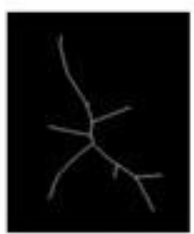

limiar 15

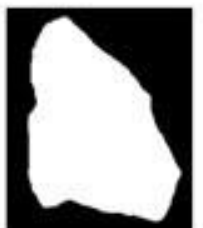

(b)

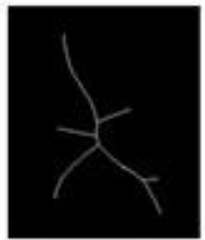

limiar 20

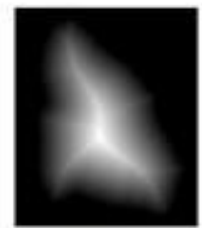

(c)

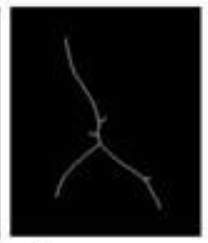

limiar 25

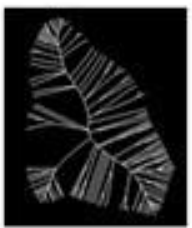

limiar 1

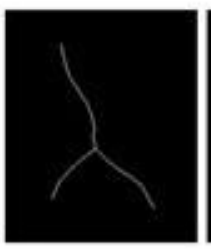

limiar 30

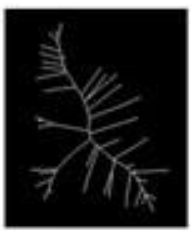

limiar 5

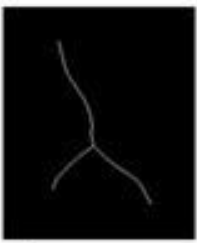

limiar 35

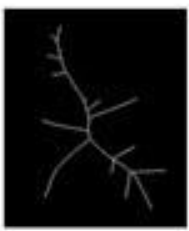

limiar 10

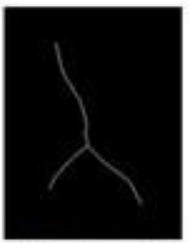

limiar 40

Figura 1. Imagem ilustrativa de: (a) partícula de agregado de basalto, (b) imagem binária da partícula, (c) imagem da Transformada Distância da partícula, e esqueletos com vários níveis de detalhamento (limiares)

tório do esqueleto para representar a forma da partícula. Estão ilustrados, nesta Figura, esqueletos formados quando são utilizados valores de limiar de 1, 5, 10, 15, 20, 25, 30, 35 e 40.

\section{PROGRAMA EXPERIMENTAL}

\subsection{Aquisição das Imagens}

Imagens de dois tipos diferentes de agregados foram obtidas, sendo 100 partículas de agregado de basalto e 100 partículas de agregado de seixo. Um scanner de base plana e de alta definição (4800 dpi) foi utilizado para a aquisição das imagens. As partículas do agregado foram dispostas sobre o scanner, apoiando cada partícula sob seu maior eixo, ou seja, eixo de maior estabilidade. As imagens foram obtidas com resolução de 300 dpi em um ambiente completamente escuro, evitando, dessa maneira, a interferência de outras fontes de luz. Foi observado que, nestas condições, as bordas do agregado ficavam bem definidas. Um exemplo de imagem obtida pode ser visto na Figura 2. Após a aquisição, as imagens foram segmentadas, pré-processadas para eliminar ruídos e, então, obtidas suas imagens binárias.

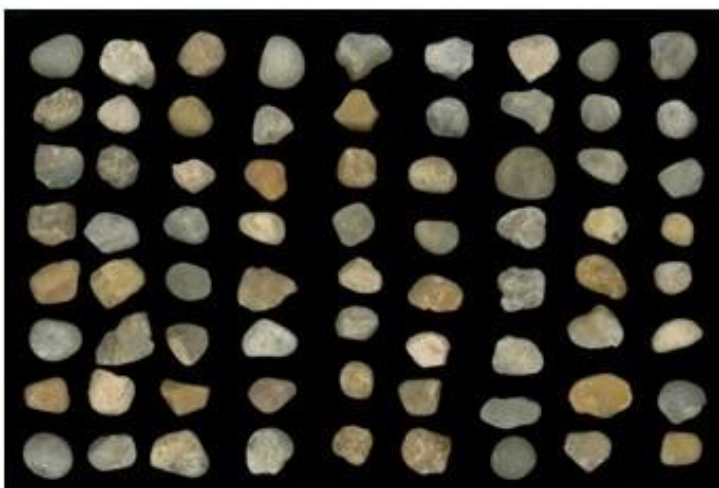

Figura 2. Imagem do agregado de seixo obtida através do scanner em ambiente completamente escuro

\subsection{Esqueletização Multiescala}

O esqueleto do objeto foi obtido por meio da Transformada Distância, considerando a distância Euclidiana en- tre cada ponto interior da imagem e sua borda mais próxima. Foram utilizados valores de limiares de 1 a 40, variando de cinco em cinco (Figura 1). A utilização da variação de limiares permite encontrar um nível de detalhamento satisfatório para representar a forma da partícula através do controle do grau de detalhamento dos esqueletos (Costa e Estrozi, 1999). Esqueletos obtidos para maiores valores de limiares tendem a suavizar os detalhes da forma e, inversamente, valores menores de limiares tendem a representar mais detalhes.

Os valores das maiores distâncias do centro do agregado com relação às bordas, denominadas de eixo médio, foram detalhadas por histogramas. Estimou-se a média e o desvio padrão das distâncias sobre cada ponto do eixo médio da partícula. Foram calculados também o número de ramos e o comprimento do maior ramo detectado no esqueleto. Estas características extraídas das imagens foram denominadas de descritores. Os descritores foram então utilizados como dados de entrada para extrair conhecimento das amostras, de modo a promover a classificação dos agregados quanto à forma.

\subsection{Aprendizado de Máquina}

Aprendizado de máquina é uma área da inteligência artificial que consiste no desenvolvimento e na utilização de técnicas computacionais capazes de extrair conhecimento a partir de amostras de dados (Witten e Frank, 2005). Nesse contexto, o conhecimento refere-se à informação armazenada ou aos modelos utilizados para interpretar, predizer e responder adequadamente ao problema considerado (Costa e Cesar Jr., 2001).

Os algoritmos computacionais que executam técnicas de aprendizado de máquina, comumente chamados de classificadores, têm como objetivos encontrar e descrever padrões a partir dos dados obtidos do ambiente. O aprendizado pode ser realizado de diferentes formas, de acordo com um paradigma. Exemplos de paradigmas são: simbólico, estatístico, baseado em instâncias, conexionista e genético. A tarefa de aprender através desses paradigmas consiste em escolher ou adaptar os parâmetros de representação do modelo. Independentemente do paradigma, os algoritmos de aprendizado têm a tarefa principal de aprender um modelo a partir do ambiente e manter esse modelo consistente de 
modo a atingir os objetivos de sua aplicação. Explicar os dados e fazer predições são objetivos comuns da aplicação desses algoritmos. Neste trabalho, os algoritmos IBk, Naive Bayes e J48 foram avaliados para o problema de classificação. Tais classificadores são largamente adotados e produzem com frequência os melhores resultados em relação ao desempenho de classificação.

$\mathrm{O}$ algoritmo IBk segue o modelo de aprendizado baseado em instâncias (IBL - Instance-based learning). Nesse modelo, algoritmos de aprendizado armazenam e utilizam um conjunto de experiências de uma base (ou memória). Cada experiência, também chamada de instância, consiste em um conjunto de atributos (descritores), que podem ser classificados como atributos de entrada ou de saída. Atributos de entrada descrevem as condições em que a experiência foi observada e os atributos de saída representam o que ocorreu durante essa experiência. Durante a generalização, esses algoritmos calculam a similaridade entre uma consulta e a base de experiências, através de alguma função de distância, para determinar as experiências previamente observadas mais próximas à consulta, e assim predizer o atributo de saída para a consulta em questão. A generalização nesses algoritmos é somente iniciada quando uma nova instância necessita ser classificada. Assim, o treinamento nesses algoritmos consiste apenas no armazenamento das instâncias na base de experiências. A generalização é disparada no momento da consulta. Um método utilizado para o aprendizado baseado em memória é o k-vizinhos mais próximos (k-nearest neighbor learning) e dá origem ao algoritmo IBk, sendo que o valor de $\mathrm{k}$ define a quantidade de vizinhos a serem empregados. Esse método assume que todas as instâncias são pontos em um espaço de dimensão n. Os vizinhos mais próximos são calculados através de uma função de distância.

Para uma instância arbitrária $\mathrm{x}$ descrita como um vetor $\mathrm{a}_{1}(\mathrm{x}), \mathrm{a}_{2}(\mathrm{x}), \ldots, \mathrm{a}_{\mathrm{n}}(\mathrm{x})$, onde $\mathrm{a}_{\mathrm{i}}(\mathrm{x})$ consiste no valor do $\mathrm{i}-$ ésimo atributo de uma instância $\mathrm{x}$, adota-se a função de distância euclidiana heterogênea (Wilson e Martinez, 1997). Essa função é chamada de heterogênea por permitir valores contínuos, discretos e nominais nos atributos. Tal função, chamada de $\mathrm{E}(\mathrm{x}, \mathrm{y})$, é definida como: $E\left(x_{q}, x\right)=\sqrt{\sum_{i=1}^{n} d\left(a_{1}\left(x_{q}\right), a_{i}(x)\right)^{2}}$, onde xq é o ponto de consulta e x é uma instância na base de experiências, ambos tendo um número de componentes igual a n. A função de distância $\mathrm{d}(\mathrm{x}, \mathrm{y})$ entre dois atributos de dois pontos é definida ou como $d_{1}(x, y)$, quando empregados atributos com valores discretos, ou como $\mathrm{d}_{2}(\mathrm{x}, \mathrm{y})$, quando empregados atributos com valores contínuos: $d_{1}(x, y)=\left\{\begin{array}{c}0 \text { se } x=y \\ 1 \text { caso contrário }\end{array}\right\}$ e $d_{2}(x, y)=\|x-y\|$.

Para garantir que a distância entre atributos contínuos de uma mesma instância estejam na mesma faixa (entre $0 \mathrm{e}$ 1), alguma forma de normalização é necessária. A mais comum é a normalização linear. Sendo $\max _{\mathrm{xi}} \mathrm{o}$ valor máximo observado na base de experiência para o atributo xi e $\min _{\mathrm{xi}}$ o valor mínimo observado para o mesmo atributo, a função de normalização linear $\mathrm{h}(\mathrm{xi})$ é definida como: $h\left(x_{i}\right)=x_{i}-\min _{x_{i}} / \max _{x_{i}}-\min _{x_{i}}$. A normalização permite que os atributos das instâncias estejam na mesma escala durante o cálculo da distância. A normalização é efetuada nas instâncias no momento em que são inseridas na base de experiências.

No aprendizado baseado nos k-vizinhos mais próximos, a função objetivo pode ser um valor discreto ou contínuo. A operação da técnica para funções objetivo com valores discretos (que se aplica ao problema em questão), é expressa na forma $f: R^{n} \rightarrow V^{\prime}$, onde $\mathrm{V}$ é um conjunto finito $\{\mathrm{v} 1, \ldots, \mathrm{vs}\}$. O valor $f^{\prime}\left(x_{q}\right)$ retornado como uma estimativa da função objetivo $f\left(x_{q}\right)$ é o valor mais comum de f entre os k exemplos de treinamento próximos a

O Treinamento no método baseado em instâncias consiste em adicionar cada amostra de dados $(x, f(x))$ à base de experiências. A classificação é realizada, dado um ponto de consulta $x_{q}$ a ser classificado e um conjunto $x_{1} \ldots x_{k}$ de $k$ instâncias da base de experiências mais próximas ao ponto , que compõem um conjunto de instâncias vizinhas $V$, retornando $f^{\prime} \leftarrow \operatorname{argmax}_{v \in V} \sum_{i=1}^{k} \delta(v, f(x))$, onde $\delta(a, b)=1$, se $a=b$, ou 0 , no caso contrário.

Naive Bayes é um classificador estatístico, baseado no teorema de Bayes, chamado de ingênuo (naive) por assumir que os atributos são condicionalmente independentes. Assim, um problema de aprendizado supervisionado, como o em questão, de aproximar uma função alvo $\mathrm{f}: \mathrm{X} \rightarrow \mathrm{C}$ torna-se equivalente a estimar $\mathrm{P}(\mathrm{X} \mid \mathrm{C})$, onde $\mathrm{C}$ corresponde a classe do agregado (forma) e X a instância (descritores). O classificador Naive Bayes combina o modelo de probabilidade condicional de Bayes com uma regra de decisão. A regra de decisão consiste em escolher a hipótese mais provável (que corresponde a forma provável do agregado). Quando uma nova instância x é apresentada, o classificador atribui a classe mais provável conforme a seguinte função: $f(x)=\arg _{\text {max }_{c j \in C}} P\left(c_{i}\right) \prod_{I} P\left(a_{i} / c_{j}\right)$, onde $\mathrm{a}_{\mathrm{i}}$ descreve o iésimo atributo da instância e $c_{j}$ corresponde a j-ésima classe (forma do agregado). Assim, a classificação Naive Bayes é realizada por meio da construção de uma hipótese pela estimativa de diferentes $\mathrm{P}(\mathrm{ci})$ e de $\mathrm{P}(\mathrm{ai} \mid \mathrm{cj})$ baseando-se nas frequências e nos dados de treinamento (conjunto dos valores de descritores obtidos por meio da esqueletização).

Por fim, o classificador J48 segue o modelo de árvores de decisão. Árvores de decisão permitem derivar regras de classificação, obtidas pelos caminhos de um nó a outro da árvore saindo do nó raiz (atributo de maior relevância) até os nós folha (atributo de interesse). Assim, para verificar os padrões obtidos, basta percorrer o trajeto do nó raiz até um nó folha nesta árvore. O classificador J48 constrói árvores de decisão empregando o conceito de entropia da informação. O algoritmo atua escolhendo o atributo no conjunto de dados que mais efetivamente separa os dados de treinamento em subconjuntos. $O$ critério de separação é a diferença nos valores de entropia. A Figura 3 ilustra um fragmento de árvore de decisão para classificação de forma. Nesse caso, o descritor "media", de maior entropia, é a raiz para a decisão. Caso o seu valor seja menor ou igual a 1,44, consulta-se o valor do descritor "qtde" (quantidade de ramos). Se essa quantidade for menor ou igual a 25 , classificase o agregado como seixo (representado pelo valor 1 ). Se a quantidade for maior que 25 , deve-se observar novamente a média e assim por diante, até atingir o final (folhas) da árvore de decisão. 
GOUVEIA, L.T.; STELLE, F.E.; SENGER, L.J.; FILHO, E.A.M.

Tabela 1. Grau de esfericidade dos agregados, variância da média e classificação de forma

\begin{tabular}{llll}
\hline Agregado & Grau de esfericidade & Variância da média & Classificação de forma \\
\hline Seixo & 0,88 & 0,011 & Esférica \\
Basalto & 0,78 & 0,005 & Subalongada \\
\hline
\end{tabular}

\begin{tabular}{|l|l|}
\hline media $<=1.44$ \\
qtde $<=25: 1$ \\
qtde $>25$ \\
$|\quad|$ media $<=1.36: 0$ \\
$|\quad| \quad$ media $>1.36$ \\
$\mid$ | variancia $<=114: 1$ \\
$\mid$ | variancia $>114: 0$
\end{tabular}

Figura 3. Exemplo de árvore de decisão J48 obtida para o problema de classificação de forma

\subsection{Método Convencional de Análise de Forma}

Imagens das 100 partículas de agregado de basalto e 100 imagens de agregado de seixo foram examinadas uma a uma e comparadas com as imagens de forma de agregados da tabela de Rittenhouse. A tabela de Rittenhouse classifica a esfericidade numericamente de 0,45 até 0,97 (Rittenhouse, 1943). Quanto mais próximo de 1 for o grau de esfericidade, mais a forma da partícula se aproxima de uma esfera. Este é um método de análise por comparação visual convencional que, apesar de laborioso e demorado, foi utilizado com o objetivo de verificar a adequabilidade do método de esqueletização multiescala proposto para a avaliação da forma dos agregados.

\section{APRESENTAÇÃO E ANÁLISE DOS RESULTADOS}

\subsection{Método Convencional de Análise Visual}

$\mathrm{Na}$ Tabela 1 estão apresentadas as médias das formas das partículas dos dois tipos de agregado, basalto e seixo, obtidas através da tabela de Rittenhouse, assim como as variâncias e a classificação de forma.

Para fazer uma classificação da forma dos agregados com base nos resultados obtidos do grau de esfericidade da tabela de Rittenhouse, optou-se por estabelecer limites de valores correspondentes à forma, uma vez que a classificação de Rittenhouse é baseada em valores quantitativos e não qualitativos de grau de esfericidade. Os limites foram estabelecidos conforme os níveis de esfericidade (arredondamento) e de alongamento, como mostra a Figura 4.

Os resultados mostram que as partículas de basalto são, em geral, subalongadas, com grau de esfericidade médio de 0,78. Já as partículas do agregado de seixo são, em geral, arredondadas com grau de esfericidade médio de 0,88 . As médias das formas foram analisadas através do método estatístico teste $\mathrm{t}$, considerando um nível de confiança de $95 \%$.

\subsection{Esqueletização Multiescala}

\subsubsection{Seleção dos descritores}

Os descritores utilizados para detectar as diferenças entre as formas dos agregados foram: média e variância dos eixos médios; número de ramos dos esqueletos; comprimento do maior ramo do esqueleto; e a razão da área da partícula pelo comprimento do maior ramo (esfericidade).
Duas análises estatísticas foram realizadas para a seleção dos melhores descritores, uma utilizando um teste estatístico simples, o teste $\mathrm{T}$ e outra utilizando análise de componentes principais (PCA). O teste T é um teste estatístico paramétrico que emprega conceitos estatísticos para rejeitar ou não uma hipótese nula quando a estatística do teste segue uma distribuição t de Student. PCA é também um procedimento estatístico, mas que emprega uma transformação ortogonal para converter um conjunto de observações de variáveis possivelmente correlacionadas a um conjunto de valores de variáveis linearmente não correlacionadas chamadas de componentes principais. O número de componentes principais é menor ou igual ao número de variáveis originais. Os componentes principais são ditos ortogonais por serem os autovalores de uma matriz de covariância, que é simétrica.

As análises feitas a partir dos valores estimados de cada descritor, em vários níveis de detalhamento (limiares), mostram que os descritores foram sensíveis para detectar diferenças de forma entre os agregados, com 95\% de confiança, considerando tanto o teste estatístico t quanto o PCA. Os resultados indicaram que todos os descritores são importantes para a classificação dos agregados, pois foram capazes de diferenciar a forma dos agregados de basalto e de seixo, realmente distintas, que apresentaram graus de esfericidade de 0,78 (subalongado) e 0,88 (esférico), respectivamente. Portanto, todos os descritores foram considerados para a próxima fase de análise de adequabilidade de cada descritor para detecção da forma.

\subsubsection{Análise de adequabilidade dos descritores e dos limiares- Método Visual Gráfico}

Como todos os descritores utilizados se mostraram promissores para diferenciar formas dos agregados, estes descritores foram pareados e gráficos bidimensionais foram projetados com o objetivo de promover uma análise visual de adequabilidade dos descritores em função dos limiares. Um exemplo desta análise pode ser visto na Figura 5, onde estão apresentados como descritores, o número de ramos e a média dos raios, com limiares 5 (Figura 5 (a)) e 20 (Figura 5 (b)).

Pode-se notar na Figura 5 (a) e (b), que, para um mesmo par de descritores houve diferenças de separação entre classes de agregados devido ao uso de diferentes limiares. É possível observar a ocorrência de melhor separação entre classes de forma dos agregados ao utilizar o limiar 5 se comparado ao limiar 20. Esse fato foi verificado para todos os descritores, ou seja, os esqueletos formados com limiar 5 apresentaram um nível de detalhamento de forma que melhor propiciou a diferenciação entre as formas dos agregados estudados.

Quanto a adequabilidade dos descritores, pode-se verificar que os descritores que aparentemente promoveram a melhor separação entre classes de agregados foram o número de ramos e a média dos raios e; a variância e o número de ramos, ambos com limiar 5. Já os descritores pareados 


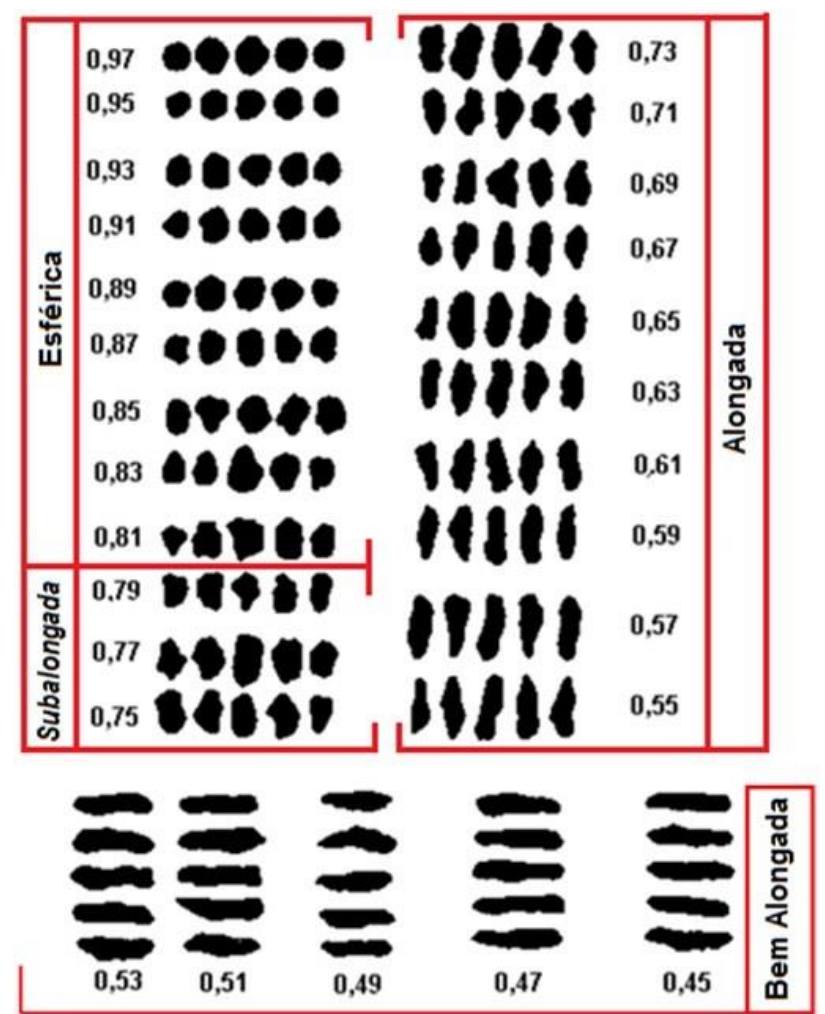

Figura 4. Classificação de forma de agregados com base nos valores de esfericidade de Rittenhouse

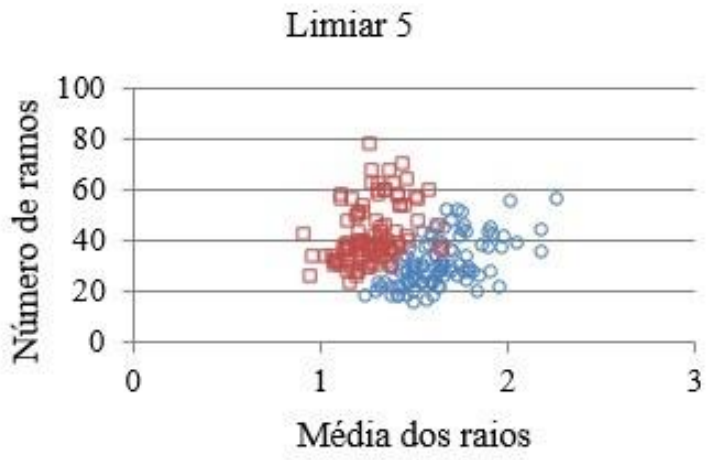

(a)

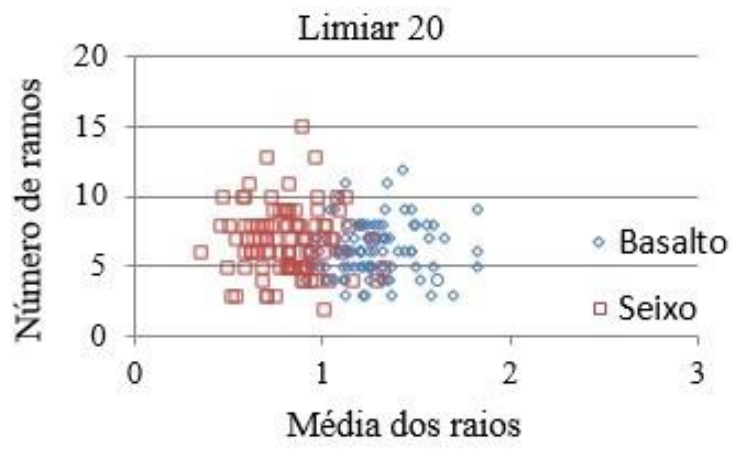

(b)

Figura 5. Exemplo de análise gráfica de descritores pareados e limiares

comprimento do maior ramo e número de ramos aparentemente não promoveram separação entre as classes.

\subsubsection{Análise de Adequabilidade dos Descritores e dos limiares - Método de Aprendizado de Máquina}

A técnica de análise visual gráfica, anteriormente citada como análise preliminar, apesar de válida, é deficiente por considerar apenas descritores dois a dois e não combinações conjuntas dos cinco descritores utilizados, além de não permitir mensurar a melhor combinação de descritores e limiares. Portanto, uma abordagem inspirada em técnicas de aprendizado de máquina é adotada de maneira a solucionar essas limitações, além de automatizar o processo de classificação, tornando-o rápido e menos suscetível a erros.

Todos os descritores foram considerados para a classificação, média dos raios, variância, número de ramos, comprimento do maior ramo e a esfericidade (razão entre o tamanho da imagem do agregado, área do agregado, pelo tamanho do maior ramo obtido na esqueletização). Assim, o conjunto final de descritores corresponde ao conjunto $\{\mathrm{M}$; $\mathrm{V}$; $\mathrm{Q} ; \mathrm{T} ; \mathrm{E}$ \}, onde $\mathrm{M}$ é o raio médio, $\mathrm{V}$ é a variância, $\mathrm{N}$ número de ramos do esqueleto, T é o tamanho do maior ramo e E corresponde a esfericidade do agregado.

$\mathrm{Na}$ Tabela 2, estão apresentados os resultados, expressos pelo índice de acerto, dos classificadores IBk, Naive Bayes e J48. Para os experimentos, foram considerados valores de vizinhança no conjunto $\{1 ; 2 ; 5 ; 10\}$ (Costa e Mutinari, 2003; Costa e Cesar Jr., 2001). O desempenho dos classificadores foi avaliado através do teste t com um nível de confiança igual a 95\%. O melhor valor absoluto foi obtido para um valor de limiar igual a 5 , que produz um índice de acerto na classificação igual a 96,85\%. O limiar 1, com maior nível de detalhamento, produziu esqueletos que foram afetados por pequenos detalhes de borda, incluindo a rugosidade do agregado, o que possivelmente tenha contribuído para diminuir os acertos de classificação em relação ao limiar de 5. Já com o limiar 5, foram formados esqueletos ricos em detalhes e suficientes para promover a melhor classificação dos agregados quanto à forma. Os demais limiares apresentaram, de uma maneira geral, pior nível de acerto de classificação quanto maior o limiar utilizado. 
GOUVEIA, L.T.; STELLE, F.E.; SENGER, L.J.; FILHO, E.A.M.

Tabela 2. Resultados do índice de acerto, dos classificadores IBL, NaiveBayes e J48 Classificadores

\begin{tabular}{lllllll}
\hline Limiar & $I B k(k=1)$ & $I B k(k=2)$ & $I B k(k=5)$ & $I B k(k=10)$ & $N$. Bayes & $J 48$ \\
\hline 01 & 87,25 & 87,25 & 89,65 & 89,30 & 85,55 & 85,45 \\
05 & $\mathbf{9 6 , 5 0}$ & $\mathbf{9 6 , 5 0}$ & $\mathbf{9 6 , 8 5}$ & $\mathbf{9 5 , 5 5}$ & 90,45 & $\mathbf{9 4 , 2 5}$ \\
10 & 94,40 & 94,40 & 94,30 & 94,45 & 89,35 & 91,60 \\
15 & 89,40 & 89,40 & 93,10 & 92,35 & 86,35 & 92,85 \\
20 & 86,25 & 86,25 & 89,70 & 90,00 & 83,65 & 91,20 \\
25 & 85,90 & 85,90 & 88,95 & 89,10 & 82,30 & 90,75 \\
30 & 87,45 & 87,45 & 86,70 & 85,75 & 79,15 & 88,25 \\
35 & 85,85 & 86,40 & 88,25 & 86,75 & 77,50 & 88,30 \\
40 & 88,30 & 88,30 & 86,50 & 86,25 & 79,90 & 84,80 \\
\hline
\end{tabular}

Dentre os classificadores avaliados, o melhor resultado foi obtido para o classificador IBk, sendo que o melhor valor absoluto de acerto foi obtido com o valor de vizinhança igual a 5. Apesar de obter o melhor valor absoluto, o teste $\mathrm{t}$ revelou que não há diferença significativa entre os resultados obtidos com valores diferentes de vizinhança. $\mathrm{O}$ teste $t$ revelou também que o classificador IBk apresenta um desempenho superior ao algoritmo Naive Bayes e similar ao algoritmo J48, demonstrando ser um classificador adequado para classificação de forma do agregado.

\section{CONCLUSÕES}

Os resultados demonstram que a análise de forma utilizando esqueletização multiescala é promissora, pois as duas classes de agregados analisadas, basalto e seixo, formaram esqueletos bem diferenciados. Tais diferenças de forma foram detectadas através dos descritores de forma utilizados.

Os descritores utilizados foram média dos raios (M), variância $(\mathrm{V})$, número de ramos $(\mathrm{N})$, comprimento do maior ramo $(\mathrm{T})$ e a esfericidade (E). Os resultados da avaliação da adequabilidade dos descritores para a classificação da forma mostram que todos os descritores utilizados foram considerados importantes para a classificação. Resultados piores de classificação foram encontrados quando realizados experimentos com a remoção de alguns descritores. Portanto, o conjunto final de descritores considerados adequados para classificação da forma do agregado corresponde ao conjunto $\{\mathrm{M} ; \mathrm{V} ; \mathrm{Q} ; \mathrm{T} ; \mathrm{E}\}$.

O nível de detalhamento dos esqueletos considerado mais adequado, numa abordagem multiescala, foi o de limiar igual a 5, que produziram esqueletos ricos em detalhes e suficientes para promover a melhor classificação dos agregados. Os demais limiares apresentaram, de uma maneira geral, pior nível de acerto de classificação quanto maior o limiar utilizado.

Foram utilizados vários algoritmos de classificação, sendo o IBk o mais adequado dentre os utilizados, pois este apresentou um índice de acerto próximo a 97\%. Este elevado índice de acerto demonstra a robustez do método de esqueletização para a classificação dos agregados quanto à forma. O método de esqueletização se mostra muito promissor para análise e classificação da forma dos agregados, no entanto, mais testes devem ser realizados com um número maior de amostras.

\section{AGRADECIMENTOS}

Lilian Tais de Gouveia e Luciano José Senger agradecem à CNPQ pelo financiamento do projeto. Fernanda Elisa Stelle e Edson Aparecido Martins Filho agradecem à Fundação Araucária pelo apoio financeiro sob forma de bolsa de Iniciação Científica.

\section{REFERÊNCIAS}

Barrett, P. J. (1980) Sedimentology, 27, p. 291-303.

Bessa, I. S. (2012) Avaliação do Processamento Digital de Imagens como Ferramenta para Caracterização de Agregados e Misturas Asfálticas. Dissertação (Mestrado), Universidade Federal do Ceará, Fortaleza.

Bowman, E. T.; K. Soga, K. e T. W. Drummond (2000) Particle shape characterisation using Fourier analysis. Relatório técnico, Cambridge University Engineering Department CUED/D-Soils/TR315. DOI: 10.1680/geot.2001.51. 6.545

Chandan, C.; K. Sivakumar; E. Masad e T. Fletcher (2004) Application of imaging techniques to geometry analysis of aggregate particles. Journal of Computing in Civil Engineering, p. 75 - 82. DOI: dx.doi.org/10.1061/(ASCE)08873801(2004)18:1(75)

Costa, L. d. F. e L.F. Estrozi (1999) Multiresolution shape representation without border shifting, Electron. Lett, v. 35, n. 21, p. 1829-1830. DOI: 10.1049/el: 19991262

Costa, L. d. F. e R. M. Cesar Jr. (2001) Shape analysis and classification: Theory and practice, CRC, Boca Raton, Fla.

Costa, L. d. F. e G. Mutinari (2003) Characterizing width uniformity by wave propagation. Physical Review, E 68. DOI: dx.doi.org/10.1103/PhysRevE.68.056704

Fernandes, J.L; R. Roque; M. Tia; L. Casanova (2000) Evaluation of uncompacted void content of fine aggregate as a quality indicator of materials used in Superpave mixtures. Transportation Research Board. DOI: 10.3141/1723-05

Fernandes Jr., J.L.; L.T. Gouveia (2003). Limitation of Fine Aggregate Angularity Test to Predict the Behavior of Asphalt Mixtures. International Journal of Pavements, vol. 2, p. 20-28.

Fletcher, T.; C. Chandan; E. Masad; K. Sivakumar (2002)

Aggregate imaging system (AIMS) for characterizing the shape 
of fine and coarse aggregates. Transportation Research Board CD-rom.

Frazão, E. B (2002) Tecnologia de Rochas na Construção Civil. ABGE - São Paulo.

Gonzalez, R. C; R. E. Woods (2010) Processamento Digital de Imagens. Pearson - $3^{\mathrm{a}}$ edição, São Paulo, 2010.

Gouveia, L. T.; J. L. Fernandes Jr. e J. B. Soares (2007) Influência da energia de compactação no comportamento volumétrico e mecânico de misturas asfálticas. TRANSPORTES, v. 15, n. 1, p. 34-41. DOI:

hdx.doi.org/10.4237/transportes.v15i1.45.

Gouveia, L.T.; F. A. Rodrigues e L. F. Costa (2010) Multiscale Curvature Analysis of Asphaltic Aggregate Particles. Journal of Computing in Civil Engineering, v. 24, p. 506-513. DOI:dx.doi.org/10.1061/(ASCE)CP.1943-5487.0000057

Gouveia, L. T.; L. F. Costa; L. J. Senger; M. K. Albertini e R. F. Mello (2011) Entropy-Based Approach to Analyze and Classify Mineral Aggregates. Journal of Computing in Civil Engineering, ASCE, v. 25, p. 75-84. DOI: dx.doi.org/10.1061/ (ASCE) CP.1943-5487.0000071

Gouveia, L. T.; G. Arruda; F. Rodrigues, L. J. Senger e L. F. Costa (2013) Supervised Classification of Basaltic Aggregate Particles Based on Texture Properties. Journal of Computing in Civil Engineering, ASCE, v. 27, p. 177-182. DOI:

dx.doi.org/10.1061/ (ASCE) CP.1943-5487.0000212

Ketcham, R. A. e N. Shashidhar (2001) Quantitative analysis of 3-d images of asphalt concrete. Transportation Research Board CD-rom.

Kim, Y.R.; N. Kim e N. P. Khosla (1992) Effects of Aggregate Type and Gradation on Fatigue and Permanent Deformation of Asphalt Concrete. Em Effects of Aggregates and Mineral Fillers on Asphalt Mixture Performance. American Society for Testing and Materials (ASTM), Philadelphia.

Kuo, C.Y.; J. D. Frost; J. S. Lai e L. B. Wang (1996) Threedimensional image analysis of aggregate particle from orthogonal projections. Transportation Research Record, v. 1526, p. 98-103. DOI: $10.3141 / 1526-12$

Masad, E.; B. Muhunthan; N. Shashidhar e T. Harman (1999) Internal structure characterization of aspahlt concrete using image analysis. Journal of Computing in Civil Engineering, ASCE, p. 88 - 95. DOI: dx.doi.org/10.1061/(ASCE)08873801(1999)13:2(88)

Masad, E.; J. W. Button e T. Papagiannakis (2000) Fine aggregate angularity: Automated image analysis approach. Transportation Research Record, v.1721, p.66 - 72. DOI: 10.3141/1721-08

Masad, E.; Al-Rousan; J. Button; D. Little e E. Tutumluer (2005) Test Methods for Characterizing Aggregate Shape, texture, and Angularity. National Cooperative Highway Research Program, NCHRP.

Mahmoud, E. e E. Masad (2007) Experimental Methods for the Evaluation of Aggregate Resistance to Polishing, Abrasion, and Breakage. Journal of Computing in Civil Engineering, ASCE, v. 19, p. 977-985. DOI: dx.doi.org/10.1061/(ASCE)08991561(2007)19:11(977)

Senger, L.J. e L. T. Gouveia (2010) Aplicação de redes neurais ART e análise de textura para a classificação do estado de alteração de agregados minerais. Revista de Informática Teórica e Aplicada, v. 17, p. 1.

Pedrini, H. e W. R. Schwartz (2008) Análise de Imagens Digitais: Princípios, Algorítimos e Aplicações. THOMSON- São Paulo, (2008).

Prowell, B.D.; J. Zhang e E. R. Brown (2005) Aggregate Properties and Performance of Superpave Designed Hot Mix Asphalt. National Cooperative Highway Research Program, NCHRP, n. 539.

Wilson, J.D. e L. D. Klotz (1996) Quantitative Analysis of Aggregate Based on Hough Transform. Transportation Research Record, TRB, n. 1530. DOI: 10.3141/1530-14

Witten, I. A e E. Frank (2005) Data Mining: Practical Machine Learning Tools and Techniques. 2. ed. Morgan Kaufmann. 